\title{
The case for an aggressive program of dark energy probes ${ }^{1}$
}

\author{
Andreas Albrecht \\ Department of Physics, One Shields Ave.; University of California; Davis, CA 95616
}

\begin{abstract}
The observed cosmic acceleration presents the physics and cosmology communities with amazing opportunities to make exciting, probably even radical advances in these fields. This topic is highly data driven and many of our opportunities depend on us undertaking an ambitious observational program. Here I outline the case for such a program based on both the exciting science related to the cosmic acceleration and the impressive impact that a strong observational program would have. Along the way, I challenge a number of arguments that skeptics use to question the value of a strong observational commitment to this field.
\end{abstract}

Keywords: cosmic acceleration, dark energy, dark energy experiments

PACS: 98.80.-k,95.35.+d

\section{INTRODUCTION}

This is truly remarkable time to be involved in cosmology research. There are many reasons for this, but none stand out quite as dramatically as the observed cosmic acceleration (attributed in current nomenclature to the "dark energy"). In the words of the Dark Energy Task Force (DETF) [1] "most experts believe that nothing short of a revolution in our understanding of fundamental physics will be required to achieve a full understanding of the cosmic acceleration". As things stand, this revolution is being motivated both by remarkable new data sets and by exciting theoretical developments.

Many ambitious researchers in cosmology and related fields have been galvanized by these extraordinary developments. There have been a number of excellent talks at this conference on the topic of cosmic acceleration which capture some of this climate. The DETF, charged with charting a way forward with dark energy observations, received 50 thoughtful and thorough whitepapers from leaders in the field (despite the lack of any specific commitment at that time to fund future dark energy experiments).

Most of us look at these developments and are astonished at our good fortune to be part of what will surely be viewed as one of the great moments in the history of science. Indeed, since the discovery of dark energy every group that has deliberated on future directions for the field has recognized the exciting opportunities and challenges presented by the cosmic acceleration ${ }^{2}$. But a small number of negative voices continue

\footnotetext{
${ }^{1}$ Invited talk at PASCOS 07, Imperial College, July 2007

${ }^{2}$ See for example [2, 3, 4]. Since the 2006 DETF report a number of panels have recommended pursuit of specific ground and space based dark energy projects, and just since PASCOS 07 the US National Research Council's Committee on NASA's Beyond Einstein Program named a "Joint Dark Energy Mission" the top priority for that program http: //nationalacademies.org/morenews/20070907b
} 
to be heard alongside the building enthusiasm for further studies of the dark energy. To some degree this negativity may reflect the natural skepticism of scientists in the face of unbridled enthusiasm (such as the above). To the extent that that is the explanation, the skepticism is surely a good thing that will lead to a healthy debate and produce more rigorous research. On the other hand, I suspect some of the negativity is just the result of sloppy thinking and needs to be challenged and simply put to rest. Regardless of how one might attribute explanations and motivations, one purpose of this paper is to engage in this debate on a number of fronts.

Here are some illustrations of some of the negative views I am talking about. At lunch on the first day of the PASCOS 07 conference there was a lively discussion about the merits of dark energy experiments. One colleague commented

"Studies of dark energy are unlikely to be interesting because we already have a theory of dark energy."

moments later, another cosmologist declared

"Studies of dark energy are unlikely to be interesting because we have no theory of dark energy."

The two were united in their conclusion, and apparently not too bothered by subtle differences in their reasoning

One unavoidable feature of physics research is that at any given time the total amount of data is finite. That necessarily means there will be more than one theory that fits the data. In many fields, this universal fact is seen as a source of vitality. Curiosity about further resolving these degeneracies drives exciting new experiments and theoretical work. The momentous progress in physics over the last century can be seen in this light as the fundamental particles, the atomic theory of heat, quantum physics and general relativity emerged from "under the radar" of earlier data sets that were fit perfectly by more primitive theories. As was amply evident in the talks at the PASCOS 07 conference, we have good reason to look forward to similar advances as the LHC data starts coming in.

But for some reason, the fact that a given future dark energy experiment will not remove all uncertainties about the nature of dark energy seems to generate considerable angst among some physicists and astronomers ${ }^{4}$ and is sometimes given as a reason to be discouraged from even doing more experiments. As I shall quantify below (and as was also shown by others at PASCOS 07), the proposed new experiments will have an impressive impact on our knowledge of dark energy. This is all one can ever ask of a new experiment.

This paper focuses on two key areas. In the next section I review some of the thriving theoretical work that has been stimulated by the cosmic acceleration. The striking theoretical issues raised by the cosmic acceleration and the remarkable directions we have been driven in our initial attempts to understand it are the key reasons I find this topic so deeply interesting. I also believe this is why so many excellent researchers are taking risks and changing direction in their careers in order to get involved.

${ }^{4}$ See the question section of my PASCOS 07 talk for an example[5]

The case for an aggressive program of dark energy probes ${ }^{5}$

November 10, 2018 
The third section summarizes a number of results which demonstrate the tremendous impact future experiments can have on our understanding of dark energy, both on our understanding of the general properties of dark energy and in terms of constraining specific models of dark energy that are currently of interest. It is these results that demonstrate that an "aggressive program of dark energy probes" is indeed possible.

This paper is based on my talk at the PASCOS 07 meeting at Imperial College. My slides and a video of the talk are available online[5]. This online material as well as my "Origins of Dark Energy" talk[6] and related papers[7, 8] are a good source for the technical material on which this paper is based. My goal here is to assemble some key arguments in a concise form. Readers seeking more details should refer to this other material.

\section{DARK ENERGY SCIENCE}

\section{Two types of acceleration}

At the start of the era of observations of cosmic acceleration the cosmology community was familiar with two possible sources of cosmic acceleration. These two types of acceleration were sufficiently established that they could both be found in textbooks.

Cosmological constant: The cosmological constant arises as an "extra" constant term in Einstein's equations. A FRW universe with a cosmological constant with value $\Lambda$ is equivalent to adding a matter component with density $\rho_{\Lambda} \equiv \frac{\Lambda}{8 \pi G}$ and with equation of state $p=-\rho$. This equation of state insures that $\rho_{\Lambda}$ remains constant throughout the evolution of the universe. Until the mid 1990's, a commonly held belief among particle physicists and cosmologists was that $\Lambda=0$. For many this belief was partly motivated by the fact that naively quantum fields give contributions to $\Lambda$ of order $10^{60}$ or $10^{120}$ times larger than observationally acceptable values. It was widely believed that finding some symmetry or dynamical process that sets $\Lambda$ (including contributions from quantum fields) precisely to zero was the best hope to resolve this apparent discrepancy.

Dynamical acceleration: Another widely held belief that has had growing support over the last few decades is that the universe underwent a period of cosmic inflation in the distant past. A period of cosmic inflation appears to explain many features observed in the universe today, some of which seems puzzling before the idea of inflation came along.

Today inflation is quite well understood in terms of its phenomenology, but it still has a number of unresolved foundational questions. Despite these, it is certainly clear that cosmic inflation requires a period of cosmic acceleration that cannot be described by a cosmological constant. Cosmic inflation is understood to be driven by some matter field typically called the "inflaton" which exhibits an equation of state $p=w \rho$. During inflation $w$ takes values that approach $w=-1$ but it is a requirement of inflation that strict equality does not hold. This is because inflation is fundamentally a dynamical process. Not only must the universe enter and exit inflation (leading to large variations in $w$ ) but small deviations from $w=-1$ are also necessary throughout the inflationary period in order for the mechanisms of inflation to work properly.

Today, some people (one quoted in the introduction) like to push the point of view that 
there is one superior theory of cosmic acceleration, namely the cosmological constant. It is very likely that before the late 1990's, these same people believed in cosmic inflation, and also believed $\Lambda=0$. In fact, the pioneers who started contemplating $\Lambda \neq 0$ in the 1990's [9, 10] did so precisely to prop up inflation (and the associated "CDM" cosmology) in light of data which would have otherwise been problematic. In fact [9] notes the possibility of a dynamical source of acceleration and even seems to regard it somewhat favorably over a strict cosmological constant.

So the field really has two different ideas of how cosmic acceleration can come about. One is dynamical (inflation-like) and one is not dynamical (the cosmological constant). Because of this interesting dichotomy the DETF (and many others) see the discrimination between dynamical and non-dynamical sources of cosmic acceleration as the best starting point for analyzing dark energy data (both present and future).

Note that if the data continues to be consistent with a cosmological constant it will only ever bound the possibility that there is a dynamical aspect to the cosmic acceleration. In that case one can never prove that the acceleration is absolutely constant. This should be no more troubling to a physicist than the fact that we only have bounds on the photon mass, deviations from the equivalence principle and other aspects of the physical world that many presently regard as absolute truths. The reason massless photons and the equivalence principle are seen as "truth" is that we have sufficiently high quality data to know that any corrections will be very small. The impossibility of getting an absolute result in a cosmological constant driven universe should certainly not make us timid about seeking high quality data in this area as well.

\section{Some remarkable features of accelerating cosmologies}

A key part of the science case for further study of dark energy is the very exciting nature of the theoretical developments that surround this this topic. I will highlight a few of these in this section.

Strange numbers: I've already mentioned the fact that naive quantum field theory arguments predict a discrepancy in the value of $\Lambda$ by a factor of $10^{60}$ or even $10^{120}$. In addition, most dynamical models of dark energy end up requiring a new particle associated with a so called "quintessence field" that has a mass of $10^{-31} \mathrm{eV}$ (many orders of magnitude smaller than current bounds of the photon mass). With a few noteworthy exceptions, theories of quintessence do not consider the problem of how to protect such a tiny mass from quantum corrections. Also, in general such a light particle will lead to long range forces, and most quintessence models do not have a mechanism for evading current bounds on such forces. Some dynamical models require additional tuning of parameters so that the onset of cosmic acceleration can occur in the right epoch of the universe (the "why now problem"). Theorists tend to take such features of a theory as a sign that something important is missing in our understanding.

$\Lambda$ and equilibrium: If the universe has a true (non dynamical) cosmological constant with a positive value (sufficient to account for the current acceleration, or even smaller) the future of the universe will have some striking features. The effective "cosmological constant density" $\rho_{\Lambda}$ will come to completely dominate the universe as the other types 
of matter dilute with the cosmic expansion. Under these conditions the universe will approach something called "de Sitter space", which has features which fit a (somewhat generalized) notion of equilibrium: The de Sitter space is approached asymptotically by huge fraction of all possible initial states, it has a (Hawking) temperature and an entropy. The entropy of a de Sitter space has been shown to be larger than the entropy of all other cosmological states with a non-zero cosmological constant[11].

This situation implies the following picture of cosmology in the presence of a fundamental cosmological constant: The universe is eternal, and spends most of its time in the equilibrium state described above. Cosmology as we know it must emerge from very rare large fluctuations from equilibrium. I have argued elsewhere[12] that this general picture of cosmology is much more powerful than more traditional approaches to cosmological "initial conditions". This is because the probabilities are in principle assigned to different cosmologies (or fluctuations from equilibrium) by concrete calculations based on fundamental physical laws rather than ad hoc proposals for "wavefunctions of the universe" which, far from being compelling, are notorious for generating controversy.

However, the first authors to calculate probabilities in this equilibrium picture concluded that that picture is a spectacular failure because cosmologies inconsistent with our observations were exponentially favored by their calculations[13]. Sorbo and I[12] have proposed an alternative approach to these calculations which exponentially favors the standard inflationary picture of cosmology. Deeper insights into the nature of quantum gravity are needed to learn which (if either) of the two approaches is correct.

My main point here is that considering the cosmic acceleration has driven theorists to contemplate radical new ideas about how to think about cosmology, equilibrium and initial conditions.

The string theory landscape: Andrei Linde and Renata Kallosh have given excellent talks at PASCOS 07 on the string theory landscape. I will refer you to those for details. I will just make a few brief remarks here on topics that link the string theory landscape to the main focus of my discussion.

The string theory landscape marks a radical change in thinking about fundamental physics. For many, it has replaced the old idea that the correct "theory of everything" will state the fundamental particles and their parameters and couplings in one tidy package. Instead, the string theory landscape tells us that when it comes to the physical observables that are important to us, they are chosen form an enormous collection (around $10^{1000}$ ) of string theory "vacuua" that fundamentally appear to be equally valid choices.

As Linde and Kallosh have emphasized, this dramatically different approach to fundamental physics was driven in large part by attempts to reconcile string theory with the observed cosmic acceleration. In fact, many people believe that this picture leads to a successful (anthropic[14]) account of how the cosmological constant (or more correctly, the vacuum energy) should have more or less the value needed to explain the current cosmic acceleration[15]. Some of the most enthusiastic proponents of this view have even argued informally that this success is so compelling as to undermine the need to collect further data on the cosmic acceleration.

However, as you heard in other talks at this meeting, there is more to the landscape vacuua than stating that they all fundamentally appear to be equally valid. The dynamics of a state evolving in the landscape will tend to prefer some vacuua over others. Perhaps 
even an equilibrium picture could emerge. Basically, one has to do full cosmology in the landscape before real predictions can be made. I find the work along these lines extremely interesting, but the landscape is a complicated place and we appear to be very far from a final answer. It is entirely possible that once we understand the full dynamics of the landscape the results will be inconsistent with our observations (just as was found in [13] for the $\Lambda$ case), causing a complete failure of the landscape picture. Also, the acceleration in the landscape picture is expected to be observationally indistinguishable from a cosmological constant, so any observation signal of $w \neq-1$ will rule out the landscape explanation.

The string landscape is another example where attempts to understand the cosmic acceleration have driven theory in dramatic new directions.

Other explanations: There are a variety of other approaches to explain cosmic acceleration. These include modifying Einstein gravity (see [6] for numerous talks on this subject), introducing new non-accelerating physics to explain the observations[16, 17], or "even" considering the possibility that more conventional physics produce acceleration [18] or lead to observations that we are currently misinterpreting as acceleration[19, 20].

\section{THE IMPACT OF FUTURE EXPERIMENTS}

\section{The DETF}

Based on 50 whitepapers, as well as internal expertise, the DETF developed mock data (or "data models") to represent a number of future dark energy observations. They divided these data models into stages. "Stage 2" represents data from projects that are already underway, "Stage 3" projects are medium term medium cost projects, and "Stage 4" are the larger more expensive projects currently under consideration.

The DETF considered the impact of the various projected data sets on cosmological parameters in a standard cosmological model. They parameterized the dark energy by its density today as well as a two parameter description of the equation of state parameter as it evolves with the cosmic scale factor $a$ given by $w(a)=w_{0}+w_{a}(1-a)$ (the " $w_{0}-w_{a}$ " parameterization).

The DETF considered cosmologies in which the cosmic curvature is allowed to be nonzero $\left(\Omega_{k} \neq 0\right)$. While some are happy to take $\Omega_{k}=0$ as a prior assumption, I don't believe there is a good case for doing so. For one, the main reason people like the $\Omega_{k}=0$ prior is that that value is seen as a universal prediction of cosmic inflation. However, the only reason the $\Omega_{k}=0$ universe has any chance of fitting the data is due to the presence of dark energy. Since the dark energy is so poorly understood, I feel it is a mistake to assume we know how to include it correctly, and how well it may or may not fit the $\Omega_{k}=0$ picture when things are understood more completely. Secondly, there is a very interesting subclass of inflation models that allow a small deviation from $\Omega_{k}=0$ (see for example [21]). Discriminatory power between these pictures is a valuable capability to expect from future observations.

The DETF used a figure of merit given by the inverse area in $w_{o}-w_{a}$ space constrained by a given experiment. The DETF showed that good Stage 3 data will improve 
on Stage 2 constraints in $w_{0}-w_{a}$ space by a factor of order 3, and good Stage 4 experiments will result in an order of magnitude improvement. We note that in the parameter space considered by the DETF the current data (Stage 1) does not result in significant constraints, which is why current data is typically analyzed in terms of simpler cosmological models[22].

\section{Beyond the DETF}

The DETF report gives a useful perspective on the power of future experiments, but it left a number of interesting answered questions. One question is "did the DETF miss anything important by using their particular parameterization of the dark energy?" Some researchers are also concerned that the DETF dark energy parameterization is too abstract, and would like to see estimates of the impact of future experiments on specific existing models of dark energy. In the next two sections I will report on new work (since the DETF) that answers both these questions, and helps give a more complete picture of the full power of proposed dark energy experiments. Another key "beyond DETF" question involves the subtleties of combining data sets. This is an area where improvements on the DETF analysis will certainly be important and new work[23, 24] suggests such improvements will demonstrate an even greater impact for future experiments. Those improvements are not included in the analysis below, so these results should be seen as underestimates of the true potential of future experiments.

Beyond two parameters: The $w_{0}-w_{a}$ parameterization used by the DETF allows a variety of linear functions $w(a)$. With Bernstein, I modeled $w(a)$ with a much larger number of parameters[7], resulting in stepwise constant functions $w(a)$. We used a Fisher matrix analysis that allowed us to consider an orthonormal basis of independently measured mode functions $w_{j}(a)$. An advantage of this approach is that when one increases the total number of parameters (by decreasing the step width) one simply gets an ever improving approximation to the finite number of continuous modes that are well-measured by the given experiment (most other parameterizations of $w(a)$ are highly unstable against changing the number of parameters). We argue in [7] that in the small bin limit our approach allows each experiment to show exactly what it is able to measure. The well-measured modes are chosen by the properties of the experiment and thus there is no information lost due to prior assumptions about the form of $w(a)$.

Figure 1 shows the figure of merit derived using DETF parameters (dark bars) and a suitably large number of step parameters to achieve convergence onto the well-measured modes (light bars). The different bars and panels correspond to different choices of DETF data models[7]. The upshot of [7] is that the best future data sets will measure many more than two parameters, resulting in massively higher figures of merit vs. the DETF estimates. These huge figures of merit translate directly into greater discovery power through greatly improved constraints in parameter space. However, aside from an overall rescaling of the figure of merit, the other conclusions of the DETF regarding the importance of combining techniques and the relative rankings of the different data models are unchanged. 

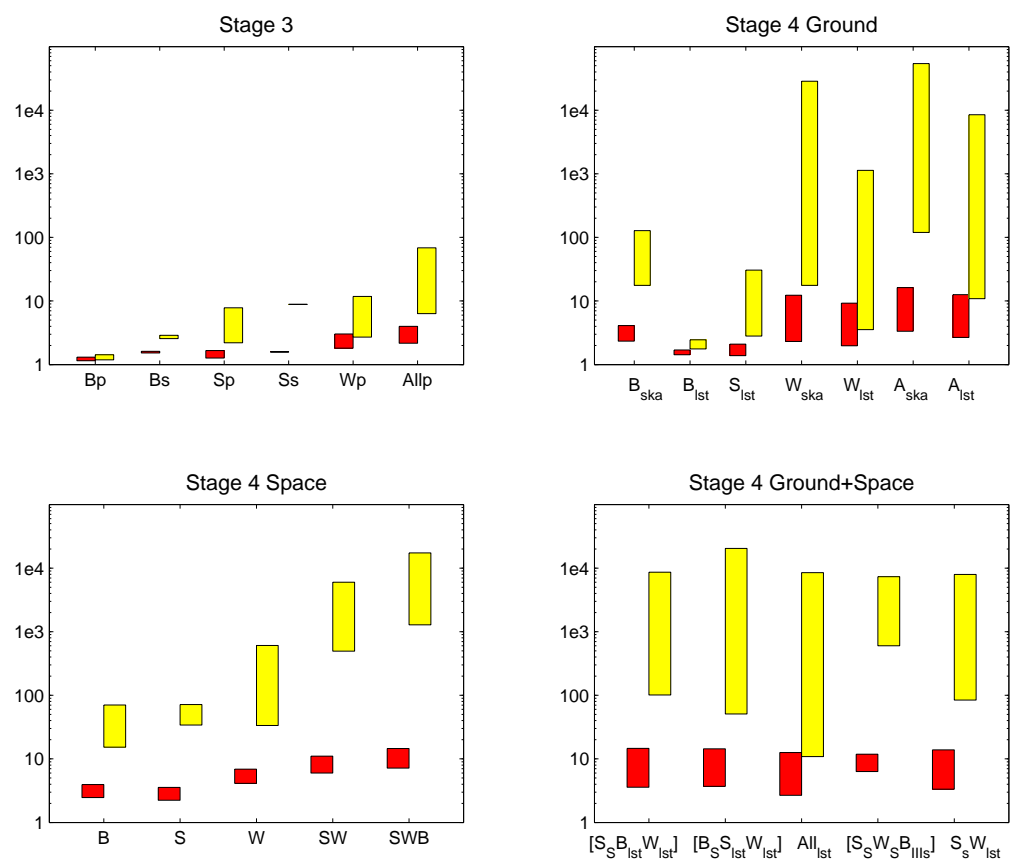

FIGURE 1. Figure 3 from [7] showing the DETF figure of merit (dark bars) and the figure of merit given by a complete set of well measured modes $w_{j}(a)$ (light bars). As detailed in [7], the higher dimensional parameter space gives a more complete picture of the ability of a given experiment to constrain dark energy properties. Good stage 4 experiments have a figure of merit many orders of magnitude higher than the DETF estimates.

Beyond abstract parameters: Theories of dark energy appear to be in a very primitive state, and are likely to change greatly before we have a deep understanding of the cosmic acceleration. To some the use of abstract parameters such as I've used above is the best approach. Basic characteristics of the dark energy can be discussed (such as whether or not it is dynamical) without dependence on a particular model that is unlikely to survive progress in this field.

Others would prefer to know if future experiments will have an impact on existing proposed explanations of dark energy. However flawed the existing explanations may turn out to be, they are what we have to work with for now and one certainly should expect proposed experiments to have an impact on these models.

To assess this point, my students and I have undertaken a number of studies of the impact of DETF data models on specific models of dark energy. You can learn more about this work in $[5,6,8]$. Here I will highlight a couple of main results.

Firstly, we found that the parameters of the specific models (i.e. parameters in the quintessence potential) were constrained to a similar degree as the DETF parameters. Typically a given model had two parameters that were constrained by cosmology, so the comparison to the two parameter DETF scheme made sense. In this picture, the higher number of parameters found in [7] shows up as discriminating power between a large variety of dark energy models (even though each tends to have a smaller number of parameters, parameterizing a limited set of functions $w(a)$ that are specific to that 


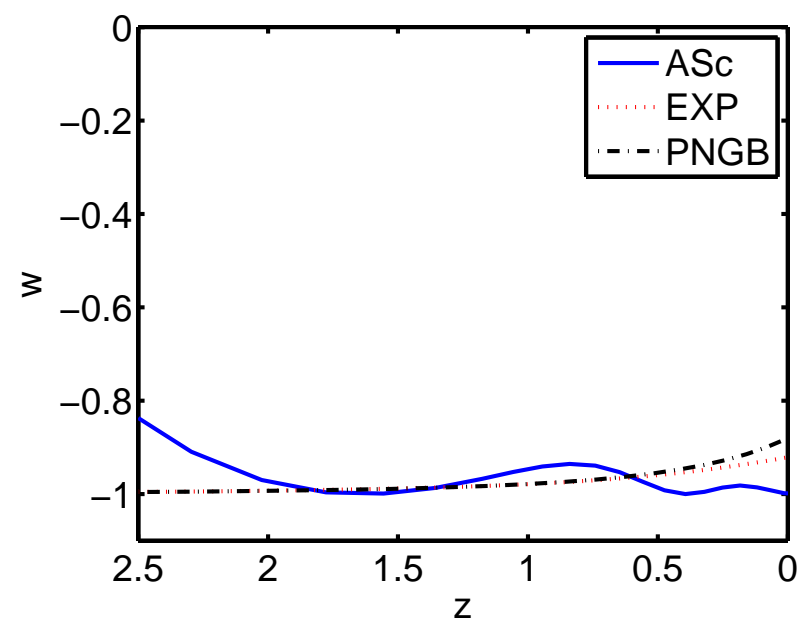

FIGURE 2. The three curves give $w(a)$ for three different models of dark energy. If the universe has chosen any of these models Stage 4 data would exclude a cosmological constant at at least four sigma .

model). $\mathrm{t}$

Figure 2 gives another illustration of the impact of future experiments. The function $w(a)$ is shown for three different quintessence models with parameters fixed at specific values. If the universe has chosen any of these three models Stage 4 data will exclude a cosmological constant to at least four sigma. For these, the discriminatory power of even the best Stage 3 experiments would be below two sigma.

\section{CONCLUSIONS AND SUMMARY}

The case for aggressive pursuit of new data on dark energy is twofold. Firstly, I have outlined how the subject of dark energy has generated very exciting and often radical new theoretical ideas. These include dramatic proposals that change how we think about equilibrium and initial conditions in cosmology and even how we formulate fundamental theories. Second, impressive new experiments are within reach that could have a tremendous impact on our understanding of dark energy. The best experiments will constrain dark energy properties orders of magnitude better than the current or medium sized future experiments, and will have the ability to strongly discriminate among and even fully eliminate popular dark energy models based on subtle variations in the equation of state.

I have outlined and challenged some of the skeptical perspectives I have heard regarding future dark energy studies. The discovery of the cosmic acceleration has caused a great upheaval in our thinking about fundamental physics and cosmology. I often sense that the skeptics are hoping this upheaval will end quickly and are grasping for arguments that will allow things to rapidly return to normal. I feel this outcome is very unlikely, and this is exactly why I find the topic so exciting.

Nature has handed us an amazing opportunity. I hope that the physics and cosmology communities have the strength to face the challenge of the cosmic acceleration head on 
and give a response that we can be proud of when people write the history of this era.

\section{ACKNOWLEDGMENTS}

I would like to thank A. Abrahamse, M. Barnard, G. Bernstein, B. Bozek, L. Sorbo, M. Yashar and the DETF members who collaborated with me on some of the work reviewed here, and B. Bozek for helpful comments on the manuscript. Also, I thank the organizers, especially Arttu Rajantie, for a really excellent conference. This work was supported in part by DOE grant DE-FG03-91ER40674 and NSF grant AST-0632901.

\section{REFERENCES}

1. A. Albrecht, et al. (2006), astro-ph/0609591.

2. M. Turner, et al. (2003), washington, USA: Nat. Acad. Pr. (2003) 206 p.

3. A. Albrecht, et al. (2005), www. interactions.org/quantumuniverse/.

4. J. A. Peacock, et al. (2006), astro-ph/0610906.

5. A. Rajantie, Pascos (2007), imperial College, July 2007. Slides and video available at http: //www.pascos 07 . org/.

6. C. Burgess, Origins of dark energy (2007), mcMaster University, May 2007. Slides available at http://origins.mcmaster.ca/darkenergy/.

7. A. Albrecht, and G. Bernstein, Phys. Rev. D75, 103003 (2007), a stro-ph/ 0608269.

8. A. Abrahamse, A. Albrecht, M. Barnard, B. Bozek, and M. Yashar (2007), three papers in preparation involving various combinations of these authors.

9. G. Efstathiou, W. J. Sutherland, and S. J. Maddox, Nature 348, 705-707 (1990).

10. L. M. Krauss, and M. S. Turner, Gen. Rel. Grav. 27, 1137-1144 (1995), a stro-ph/ 9504003.

11. G. W. Gibbons, and S. W. Hawking, Phys. Rev. D15, 2738-2751 (1977).

12. A. Albrecht, and L. Sorbo, Phys. Rev. D70, 063528 (2004), hep-th/ 0405270.

13. L. Dyson, M. Kleban, and L. Susskind, JHEP 10, 011 (2002), hep-th/ 0208013.

14. S. Weinberg, Rev. Mod. Phys. 61, 1-23 (1989).

15. R. Bousso, and J. Polchinski, JHEP 06, 006 (2000), hep-th / 0004134.

16. C. Csaki, N. Kaloper, and J. Terning, Phys. Rev. Lett. 88, 161302 (2002), hep-ph/ 0111311.

17. C. Csaki, N. Kaloper, and J. Terning, Ann. Phys. 317, 410-422 (2005), a st ro-ph/ 0409596.

18. S. Deser, and R. P. Woodard (2007), arXiv:0706.2151 [astro-ph] .

19. E. W. Kolb, S. Matarrese, and A. Riotto, New J. Phys. 8, 322 (2006), a st ro-ph/ 0506534.

20. P. Martineau, and R. Brandenberger (2005), astro-ph/ 0510523.

21. B. Freivogel, M. Kleban, M. Rodriguez Martinez, and L. Susskind, JHEP 03, 039 (2006), hep-th / 0505232.

22. L. Knox, Phys. Rev. D73, 023503 (2006), astro-ph / 0503405.

23. H. Zhan, JCAP 0608, 008 (2006), a stro-ph / 0605696.

24. M. Schneider, L. Knox, H. Zhan, and A. Connolly (2006), astro-ph/0606098. 